\title{
Well Posed Problems and Boundary Conditions in Computational Fluid Dynamics
}

CONFERENCE PAPER · JUNE 2015

DOWNLOADS

28

1 AUTHOR:

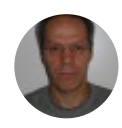

Jan Nordström

Linköping University

184 PUBLICATIONS 2,114 CITATIONS

SEE PROFILE
VIEWS

16 


\title{
Well Posed Problems and Boundary Conditions in Computational Fluid Dynamics
}

\author{
Jan Nordström* \\ Division of Computational Mathematics, Department of Mathematics, Linköping University \\ 58183 Linköping, Sweden
}

\begin{abstract}
All numerical calculations will fail to provide a reliable answer unless the continuous problem under consideration is well posed. Well-posedness depends in most cases only on the choice of boundary conditions. In this paper we will highlight this fact by discussing well-posedness of the most important equations in computational fluid dynamics, namely the time-dependent compressible Navier-Stokes equations.

In particular, we will discuss $i$ ) how many boundary conditions are required, $i i$ ) where to impose them and iii) which form they should have. The procedure is based on the energy method and generalizes the characteristic boundary procedure for the Euler equations to the compressible Navier-Stokes equations.

Once the boundary conditions in terms of $i$-iii) are known, one issue remains; they can be imposed weakly or strongly. The weak and strong imposition is discussed for the continuous case. It will be shown that the weak and strong boundary procedures produce identical solutions and that the boundary conditions are satisfied exactly also in the weak procedure.

We conclude by relating the well-posedness results to energy-stability of the numerical approximation. It is shown that the results obtained in the well-posedness analysis for the continuous problem generalizes directly to stability of the discrete problem.
\end{abstract}

\section{Introduction}

Well-posed boundary conditions are an essential ingredient in many areas of computational physics. In fluid dynamics, characteristic boundary conditions for the Euler equations have long been accepted as one way to impose boundary conditions since the specification of the ingoing characteristic variable at a boundary implies well-posedness. Often the Euler boundary conditions are used as a guidance when boundary conditions are chosen for the compressible Navier-Stokes equations. ${ }^{1-4}$ We have used this guidance before ${ }^{3,5}$ and in this paper we will refine these ideas even more, but also point out differences.

To obtain a well posed initial boundary value problem, one needs to know: i) how many boundary conditions are required, ii) where to impose them and iii) which form they should have. There are essentially two different methods to use, namely the energy method ${ }^{5}$ and the Laplace transform method. ${ }^{6}$ The number of boundary conditions and where to place them can be determined using the Laplace transform method. ${ }^{7,8}$ However, the exact form of the boundary conditions cannot be obtained; information regarding that must come from other sources. The energy method, on the other hand, provides information on all the items $i$-iii).

Throughout this paper we assume that we have unlimited access to accurate boundary data. We do not consider non-reflecting or absorbing boundary conditions ${ }^{9,10}$ even though we expect that the derived boundary conditions will perform reasonably well, provided that the corresponding data is known. Even though we focus on the compressible Navier-Stokes, it will be shown how to obtain information on i-iii) for a general time dependent partial differential equation. The procedure is based on the energy method and has clear similarities to the derivation of characteristic boundary conditions for hyperbolic problems. ${ }^{3,5}$

It has been shown that a weak imposition of boundary conditions for finite difference, ${ }^{11,12}$ finite volume, ${ }^{13,14}$ spectral element, ${ }^{15,16}$ discontinuous Galerkin ${ }^{1,17}$ and flux reconstruction schemes ${ }^{18,19}$ on summationby-parts (SBP) form lead to energy stability. We will show that the weak and strong boundary procedures

*email: jan.nordstrom@liu.se 
produce identical solutions and that the boundary conditions are satisfied exactly also in the weak procedure. We will also show that an analysis of the continuous problem with weak boundary procedures together with schemes on SBP form leads to automatic stability. No specific additional analysis of the semi-discrete problem is necessary.

\section{Preliminaries}

To set the stage for the analysis below, we consider a smooth flow situation governed by the linearized time-dependent compressible Navier-Stokes equations. The dependent variables are the density $\tilde{\rho}$, the velocity components $\tilde{u}, \tilde{v}, \tilde{w}$ in the $x, y, z$ directions and the temperature $\tilde{T}$. The tilde sign signifies the presence of dimensions and the subscript $\infty$ denotes reference values in the free stream. The equations are written in non-dimensional form using the free stream density $\tilde{\rho}_{\infty}$, the free stream velocity $\tilde{U}_{\infty}$ and the free stream temperature $\tilde{T}_{\infty}$. The shear and second viscosity coefficients $\tilde{\mu}, \tilde{\lambda}$ as well as the coefficient of heat conduction $\tilde{\kappa}$ are non-dimensionalized with the free stream viscosity $\tilde{\mu}_{\infty}$. The pressure in non-dimensional form becomes, $p=\tilde{p} /\left(\tilde{\rho}_{\infty} \tilde{U}_{\infty}^{2}\right)=\rho T /\left(\gamma M_{\infty}^{2}\right)$. Also used are

$$
M_{\infty}^{2}=\frac{\tilde{U}_{\infty}^{2}}{\gamma \tilde{R} \tilde{T}_{\infty}}, \quad \operatorname{Pr}=\frac{\tilde{\mu}_{\infty} C_{p}}{\tilde{\kappa}_{\infty}}, \quad \operatorname{Re}=\frac{\tilde{\rho}_{\infty} \tilde{U}_{\infty} \tilde{L}}{\tilde{\mu}_{\infty}}, \quad \gamma=\frac{C_{p}}{C_{v}}, \quad \varphi=\frac{\gamma \kappa}{\operatorname{Pr}}
$$

where $\tilde{L}$ is a length scale and $M, \operatorname{Pr}, \operatorname{Re}=1 / \epsilon$ and $\gamma$ are the Mach, Prandtl and Reynolds numbers and ratio of specific heats respectively. The time scale is $\tilde{L} / \tilde{U}_{\infty}$.

\section{A. The governing equations}

The three-dimensional linearized time-dependent compressible Navier-Stokes equations in non-dimensional form ${ }^{20}$ can be written

$$
V_{t}+\bar{A} V_{x}+\bar{B} V_{y}+\bar{C} V_{z}=\bar{F}_{x}+\bar{G}_{y}+\bar{H}_{z}
$$

where

$$
\bar{F}=\bar{D}_{11} V_{x}+\bar{D}_{12} V_{y}+\bar{D}_{13} V_{z}, \quad \bar{G}=\bar{D}_{21} V_{x}+\bar{D}_{22} V_{y}+\bar{D}_{23} V_{z}, \quad \bar{H}=\bar{D}_{31} V_{x}+\bar{D}_{32} V_{y}+\bar{D}_{33} V_{z} .
$$

where the subscripts $t, x, y, z$ denotes partial differentiation with respect to time and space. In (2), $V=$ $(\rho, u, v, w, T)^{T}$ is the perturbation from the constant state (denoted by an overbar) around which we linearize. The matrices related to the hyperbolic terms in (2) must be symmetric for the energy method to be applicable. ${ }^{5}$ We choose the symmetrizer

$$
S^{-1}=\operatorname{diag}\left[\frac{\bar{c}^{2}}{\sqrt{\gamma}}, \bar{\rho} \bar{c}, \bar{\rho} \bar{c}, \bar{\rho} \bar{c}, \frac{\bar{\rho}}{\sqrt{\gamma(\gamma-1) M_{\infty}^{4}}}\right],
$$

where $\bar{c}$ is the speed of sound at the constant state.

Remark 1. The three-dimensional compressible Navier-Stokes equations with 12 matrices involved (see (2) and (3)), can be symmetrized by a single matrix ${ }^{20} S$. This remarkable fact was complemented by the observation that there exist at least two different symmetrizers, based on either the hyperbolic or the parabolic terms. The symmetrizer (4) is related to the parabolic terms.

After symmetrizing (2) by multiplying it from the left with $S^{-1}$, we obtain

$$
\tilde{A}=\left(\begin{array}{ccccc}
\bar{u} & \bar{c} / \sqrt{\gamma} & 0 & 0 & 0 \\
\bar{c} / \sqrt{\gamma} & \bar{u} & 0 & 0 & \bar{c} \sqrt{\frac{\gamma-1}{\gamma}} \\
0 & 0 & \bar{u} & 0 & 0 \\
0 & 0 & 0 & \bar{u} & 0 \\
0 & \bar{c} \sqrt{\frac{\gamma-1}{\gamma}} & 0 & 0 & \bar{u}
\end{array}\right) \quad \bar{D}_{11}=\frac{\epsilon}{\bar{\rho}}\left(\begin{array}{ccccc}
0 & 0 & 0 & 0 & 0 \\
0 & 2 \bar{\mu}+\bar{\lambda} & 0 & 0 & 0 \\
0 & 0 & \bar{\mu} & 0 & 0 \\
0 & 0 & 0 & \bar{\mu} & 0 \\
0 & 0 & 0 & 0 & \bar{\varphi}
\end{array}\right)
$$




$$
\begin{aligned}
& \tilde{B}=\left(\begin{array}{ccccc}
\bar{v} & 0 & \bar{c} / \sqrt{\gamma} & 0 & 0 \\
0 & \bar{v} & 0 & 0 & 0 \\
\bar{c} / \sqrt{\gamma} & 0 & \bar{v} & 0 & \bar{c} \sqrt{\frac{\gamma-1}{\gamma}} \\
0 & 0 & 0 & \bar{v} & 0 \\
0 & 0 & \bar{c} \sqrt{\frac{\gamma-1}{\gamma}} & 0 & \bar{v}
\end{array}\right) \quad \bar{D}_{22}=\frac{\epsilon}{\bar{\rho}}\left(\begin{array}{ccccc}
0 & 0 & 0 & 0 & 0 \\
0 & \bar{\mu} & 0 & 0 & 0 \\
0 & 0 & 2 \bar{\mu}+\bar{\lambda} & 0 & 0 \\
0 & 0 & 0 & \bar{\mu} & 0 \\
0 & 0 & 0 & 0 & \bar{\varphi}
\end{array}\right) \\
& \tilde{C}=\left(\begin{array}{ccccc}
\bar{w} & 0 & 0 & \bar{c} / \sqrt{\gamma} & 0 \\
0 & \bar{w} & 0 & 0 & 0 \\
0 & 0 & \bar{w} & 0 & 0 \\
\bar{c} / \sqrt{\gamma} & 0 & 0 & \bar{w} & \bar{c} \sqrt{\frac{\gamma-1}{\gamma}} \\
0 & 0 & 0 & \bar{c} \sqrt{\frac{\gamma-1}{\gamma}} & \bar{w}
\end{array}\right) \quad \bar{D}_{33}=\frac{\epsilon}{\bar{\rho}}\left(\begin{array}{ccccc}
0 & 0 & 0 & 0 & 0 \\
0 & \bar{\mu} & 0 & 0 & 0 \\
0 & 0 & \bar{\mu} & 0 & 0 \\
0 & 0 & 0 & 2 \bar{\mu}+\bar{\lambda} & 0 \\
0 & 0 & 0 & 0 & \bar{\varphi}
\end{array}\right) \\
& \bar{D}_{12}=\bar{D}_{21}^{T}=\frac{\epsilon}{\bar{\rho}}\left(\begin{array}{ccccc}
0 & 0 & 0 & 0 & 0 \\
0 & 0 & \bar{\lambda} & 0 & 0 \\
0 & \bar{\mu} & 0 & 0 & 0 \\
0 & 0 & 0 & 0 & 0 \\
0 & 0 & 0 & 0 & 0
\end{array}\right) \quad \bar{D}_{13}=\bar{D}_{31}^{T}=\frac{\epsilon}{\bar{\rho}}\left(\begin{array}{ccccc}
0 & 0 & 0 & 0 & 0 \\
0 & 0 & 0 & \bar{\lambda} & 0 \\
0 & 0 & 0 & 0 & 0 \\
0 & \bar{\mu} & 0 & 0 & 0 \\
0 & 0 & 0 & 0 & 0
\end{array}\right) \\
& \bar{D}_{23}=\bar{D}_{32}^{T}=\frac{\epsilon}{\bar{\rho}}\left(\begin{array}{ccccc}
0 & 0 & 0 & 0 & 0 \\
0 & 0 & 0 & 0 & 0 \\
0 & 0 & 0 & \bar{\lambda} & 0 \\
0 & 0 & \bar{\mu} & 0 & 0 \\
0 & 0 & 0 & 0 & 0
\end{array}\right) \quad \tilde{V}=\left(\begin{array}{c}
\bar{c}^{2} \rho / \sqrt{\gamma} \\
\bar{\rho} \bar{c} u \\
\bar{\rho} \bar{c} v \\
\bar{\rho} \bar{c} w \\
\bar{\rho} T / \sqrt{\gamma(\gamma-1) M_{\infty}^{4}}
\end{array}\right)
\end{aligned}
$$

where $U^{T}=\left(S^{-1} V\right)^{T}, \tilde{A}=S^{-1} \bar{A} S, \tilde{B}=S^{-1} \bar{B} S, \tilde{C}=S^{-1} \bar{C} S$ and the $\bar{D}_{i j}$ 's are unchanged.

Remark 2. Note that the first row and column of the matrices $\bar{D}_{i j}$ are zero, since there are no second derivatives in the continuity equation. This makes the system (2) incompletely parabolic. ${ }^{8}$

\section{B. Well posed problems}

Roughly speaking, an initial boundary value problem is well posed if a unique solution that depends continuously on the initial and boundary data exists. Consider the following general initial boundary value problem

$$
\begin{aligned}
W_{t}+\mathcal{P} \mathcal{W} & =\mathbf{F}, \quad \mathbf{x} \in \Omega, \quad t \geq 0 \\
\mathcal{L W} & =\mathbf{g}, \quad \mathbf{x} \in \partial \Omega, \quad t \geq 0 \\
W & =\mathbf{f}, \quad \mathbf{x} \in \Omega, \quad t=0
\end{aligned}
$$

where $W$ is the solution, $\mathcal{P}$ is the spatial differential operator and $\mathcal{L}$ is the boundary operator. In this paper, $\mathcal{P}$ and $\mathcal{L}$ are linear operators, $\mathbf{F}$ is a forcing function, and $\mathbf{g}$ and $\mathbf{f}$ are boundary and initial functions, respectively. $\mathbf{F}, \mathbf{g}$ and $\mathbf{f}$ are the known data of the problem. The initial boundary value problem (10) is posed on the domain $\Omega$ with boundary $\partial \Omega$.

Definition 1. The initial boundary value problem (10) with $\mathbf{F}=\mathbf{g}=0$ is well posed if for every $\mathbf{f} \in C^{\infty}$ that vanishes in a neighborhood of $\partial \Omega$, a unique smooth solution exists that satisfies the estimate

$$
\|W(\cdot, t)\|_{\Omega}^{2} \leq K_{1}^{c} e^{\alpha_{c} t}\|\mathbf{f}\|_{\Omega}^{2}
$$

where the constants $K_{1}^{c}$ and $\alpha_{c}$ are bounded independently of $\mathbf{f}$. 
Definition 2. The initial boundary value problem (10) is strongly well posed, if it is well-posed and

$$
\|W(\cdot, t)\|_{\Omega}^{2} \leq K_{2}^{c}(t)\left(\|\mathbf{f}\|_{\Omega}^{2}+\int_{0}^{t}\left(\|\mathbf{F}(\cdot, \tau)\|_{\Omega}^{2}+\|\mathbf{g}(\tau)\|_{\partial \Omega}^{2}\right) d \tau\right)
$$

holds, where the function $K_{2}^{c}(t)$ for a limited time is bounded independently of $\mathbf{f}, \mathbf{F}$ and $\mathbf{g}$.

Remark 3. Well-posedness of (10) requires that an appropriate number of boundary conditions (number of linearly independent rows in $\mathcal{L}$ ) with the correct form of $\mathcal{L}$ (the rows in $\mathcal{L}$ have appropriate elements) is used. Too many boundary conditions means that no existence is possible, and too few that neither the estimates (11)-(12) nor uniqueness can be obtained.

Closely related to well-posedness is the concept of stability. The semi-discrete version of (10) is

$$
\begin{aligned}
\left(W_{j}\right)_{t}+\mathcal{Q} W_{j} & =\mathbf{F}_{j}, \quad \mathbf{x}_{j} \in \Omega, \quad t \geq 0 \\
\mathcal{M} W_{j} & =\mathbf{g}_{j}, \quad \mathbf{x}_{j} \in \partial \Omega, \quad t \geq 0 \\
W_{j} & =\mathbf{f}_{j}, \quad \mathbf{x}_{j} \in \Omega, \quad t=0 .
\end{aligned}
$$

The difference operator $\mathcal{Q}$ approximates the differential operator $\mathcal{P}$ and the discrete boundary operator $\mathcal{M}$ approximates $\mathcal{L} . \mathbf{F}_{j}, \mathbf{g}_{j}$ and $\mathbf{f}_{j}$ are the known data of the problem injected on the grid $\mathbf{x}_{j}=\left(x_{j}, y_{j}, z_{j}\right)$. The difference approximation (13) is a consistent approximation of (10).

Definition 3. The semi-discrete approximation (13) with $\mathbf{F}_{j}=\mathbf{g}_{j}=0$ is stable for every projection $\mathbf{f}_{j}$ of $\mathbf{f} \in C^{\infty}$ that vanishes in a neighborhood of $\partial \Omega$, if the solution $W_{j}$ satisfies the estimate

$$
\left\|W_{j}(t)\right\|_{\Omega_{h}}^{2} \leq K_{1}^{d} e^{\alpha_{d} t}\left\|\mathbf{f}_{j}\right\|_{\Omega_{h}}^{2}
$$

where the constants $K_{1}^{d}$ and $\alpha_{d}$ are bounded independently of $\mathbf{f}_{j}$ and the meshsize $h=\min _{i \neq j}\left|\mathbf{x}_{j}-\mathbf{x}_{i}\right|$.

Definition 4. The semi-discrete approximation (13 is strongly stable, if it is stable and

$$
\left\|W_{j}(t)\right\|_{\Omega}^{2} \leq K_{2}^{d}(t)\left(\left\|\mathbf{f}_{j}\right\|_{\Omega_{h}}^{2}+\int_{0}^{t}\left(\left\|\mathbf{F}_{j}(\cdot, \tau)\right\|_{\Omega_{h}}^{2}+\left\|\mathbf{g}_{j}(\tau)\right\|_{\partial \Omega_{h}}^{2}\right) d \tau\right)
$$

holds. The function $K_{2}^{d}(t)$ for a limited time is bounded independently of $\mathbf{f}_{j}, \mathbf{F}_{j}, \mathbf{g}_{j}$ and the meshsize $h$ defined above.

The definitions of well-posedness and stability above are strikingly similar. However, the bounds in the corresponding estimates need not be the same. The following definition connects the growth rates of the continuous and semi-discrete solutions.

Definition 5. Assume that (10) is well-posed with $\alpha_{c}$ in (11) and that the semi-discrete approximation (13) is stable with $\alpha_{d}$ in (14). If $\alpha_{d} \leq \alpha_{c}+\mathcal{O}(h)$ for $h \leq h_{0}$ we say that the approximation is strictly stable.

Remark 4. The norms in Definition 1-4 can be quite general but in this paper we use $\|\phi\|_{\Omega}^{2}=\int_{\Omega} \phi^{T} \phi d x d y \approx$ $\phi^{T} H \phi=\|\phi\|_{\Omega_{h}}^{2}$ and $\|\phi\|_{\partial \Omega}^{2}=\oint_{\partial \Omega} \phi^{T} \phi d s \approx \phi^{T} K \phi=\|\phi\|_{\partial \Omega_{h}}^{2}$. The matrices $H$ and $K$ define appropriate quadrature rules and $\phi$ is a smooth function. More details on the definitions above are given in. ${ }^{6}$

\section{The continuous problem}

The initial boundary value problem we will consider in this paper is obtained by adding on the boundary and initial conditions to the symmetrized version of $(2)$

$$
\begin{aligned}
& U_{t}+\bar{A} U_{x}+\bar{B} U_{y}+\bar{C} U_{z}=\bar{F}_{x}+\bar{G}_{y}+\bar{H}_{z}, \quad(x, y, z) \in \Omega, \quad t \geq 0 \\
& H U=g, \quad(x, y, z) \in \delta \Omega, \quad t \geq 0 \\
& U=f, \quad(x, y, z) \in \Omega, \quad t=0 .
\end{aligned}
$$

The solution and the matrices in (16) are given by (3)-(9) above. The formulation (16) is used for strong imposition of boundary conditions. When imposing the boundary conditions weakly we consider

$$
\begin{aligned}
U_{t}+\bar{A} U_{x}+\bar{B} U_{y}+\bar{C} U_{z} & =\bar{F}_{x}+\bar{G}_{y}+\bar{H}_{z}+L(\Sigma(H U-g)) & & (x, y, z) \in \Omega, \quad t \geq 0 \\
U & =f, & & (x, y, z) \in \Omega, \quad t=0
\end{aligned}
$$


In (17), $L$ is a lifting operator ${ }^{25,26}$ defined for smooth vector functions $\phi, \psi$ by $\int_{\Omega} \phi^{T} L(\psi) d x d y d z=$ $\oint_{\partial \Omega} \phi^{T} \psi d s$ and $\Sigma$ is an appropriate penalty matrix. The first task is now to determine the boundary operator $H$ such that (16) is well posed.

\section{A. The energy method}

Let the energy norm be defined as $\|U\|^{2}=\int_{\Omega} U^{T} U d x d y d z$. The energy method is applied to (16) by multiplying with $U^{T}$ and integrating over the domain $\Omega$. Gauss' theorem and integration by parts leads to

$$
\|U\|_{t}^{2}+2 D I_{c}=B T
$$

where

$$
D I_{c}=\int_{\Omega}\left[\begin{array}{l}
U_{x} \\
U_{y} \\
U_{z}
\end{array}\right]^{T}\left[\begin{array}{lll}
\bar{D}_{11} & \bar{D}_{12} & \bar{D}_{13} \\
\bar{D}_{21} & \bar{D}_{22} & \bar{D}_{23} \\
\bar{D}_{31} & \bar{D}_{32} & \bar{D}_{33}
\end{array}\right]\left[\begin{array}{c}
U_{x} \\
U_{y} \\
U_{z}
\end{array}\right] d x d y d z, \quad B T=-\oint_{\partial \Omega} U^{T} A U-2 U^{T} F d s .
$$

In (19), $d s=\sqrt{d x^{2}+d y^{2}+d z^{2}}, \hat{n}=\left(n_{1}, n_{2}, n_{3}\right)^{T}$ is the outward pointing unit normal on $\partial \Omega$, and

$$
A=n_{1} \tilde{A}+n_{2} \tilde{B}+n_{3} \tilde{C}, \quad F=n_{1} \bar{F}+n_{2} \bar{G}+n_{3} \bar{H} .
$$

Due to the incompletely parabolic character of the problem, we consider the following block structure of vectors and matrices in (19)

$$
U=\left[\begin{array}{l}
U_{1} \\
U_{2}
\end{array}\right], \quad F=\left[\begin{array}{c}
0 \\
F_{2}
\end{array}\right], \quad A=\left[\begin{array}{ll}
A_{11} & A_{12} \\
A_{12}^{T} & A_{22}
\end{array}\right] .
$$

In (21), $U_{1}$ is a scalar, $U_{2}$ and $F_{2}$ are four components long, $A_{11}$ is a scalar, $A_{12}$ is a $1 \times 4$ matrix and $A_{22}$ is a $4 \times 4$ matrix. With these notations we can write the quadratic form in (19) as

$$
U^{T} A U-2 U^{T} F=\left[\begin{array}{c}
U_{1} \\
U_{2} \\
F_{2}
\end{array}\right]^{T}\left[\begin{array}{ccc}
A_{11} & A_{12} & 0 \\
A_{12}^{T} & A_{22} & -I \\
0 & -I & 0
\end{array}\right]\left[\begin{array}{c}
U_{1} \\
U_{2} \\
F_{2}
\end{array}\right]
$$

where $I$ is the $4 \times 4$ identity matrix.

It is straightforward ${ }^{21}$ to show that the dissipation term on the left-hand-side in (18) is positive semidefinite. Consequently, for well-posedness it remains to bound $B T$ on the right-hand-side with a minimal number of boundary conditions. ${ }^{6}$ One needs to know $i$ ) how many boundary conditions are required, $i i$ ) where on $\partial \Omega$ to impose them and iii) which form they should have. Too many boundary conditions means no existence, and too few that neither an estimate nor uniqueness can be obtained.

\section{B. The number and position of the boundary conditions}

By rotating the boundary matrix in (19) to block diagonal form ${ }^{5}$ we obtain

$$
B T=-\oint_{\delta \Omega}\left[\begin{array}{l}
w_{1} \\
w_{2} \\
w_{3}
\end{array}\right]^{T} R^{T}\left[\begin{array}{ccc}
A_{11} & A_{12} & 0 \\
A_{12}^{T} & A_{22} & -I \\
0 & -I & 0
\end{array}\right] R\left[\begin{array}{l}
w_{1} \\
w_{2} \\
w_{3}
\end{array}\right] d s=-\oint_{\delta \Omega}\left[\begin{array}{c}
w_{1} \\
w_{2} \\
w_{3}
\end{array}\right]^{T}\left[\begin{array}{ccc}
A_{11} & 0 & 0 \\
0 & \tilde{A}_{22} & 0 \\
0 & 0 & -\left(\tilde{A}_{22}\right)^{-1}
\end{array}\right]\left[\begin{array}{l}
w_{1} \\
w_{2} \\
w_{3}
\end{array}\right] d s
$$

where

$$
\left[\begin{array}{l}
w_{1} \\
w_{2} \\
w_{3}
\end{array}\right]=R^{-1}\left[\begin{array}{l}
U_{1} \\
U_{2} \\
F_{2}
\end{array}\right]=\left[\begin{array}{c}
U_{1}+\left(A_{11}\right)^{-1} A_{12} U_{2} \\
U_{2}-\left(\tilde{A}_{22}\right)^{-1} F_{2} \\
F_{2}
\end{array}\right], \quad \tilde{A}_{22}=A_{22}-A_{12}^{T}\left(A_{11}\right)^{-1} A_{12} .
$$

Note that the rotation above requires that $A_{11}$ is nonsingular. 
Since $\tilde{A}_{22}=\tilde{A}_{22}^{T}$ we can write $\tilde{A}_{22}=X \Lambda_{22} X^{T}$ where $\Lambda_{22}=\operatorname{diag}\left(\Lambda_{22}^{+}, \Lambda_{22}^{-}\right)$and $X=\left[X_{+}, X_{-}\right]$contain the positive and negative eigenvalues and the corresponding eigenvectors respectively. By using this eigendecomposition of $\tilde{A}_{22}$, we obtain

$$
B T=-\oint_{\delta \Omega}\left[\begin{array}{c}
w_{1} \\
X_{+}^{T} w_{2} \\
X_{-}^{T} w_{2} \\
X_{+}^{T} w_{3} \\
X_{-}^{T} w_{3}
\end{array}\right]^{T}\left[\begin{array}{ccccc}
A_{11} & 0 & 0 & 0 & 0 \\
0 & \Lambda_{22}^{+} & 0 & 0 & 0 \\
0 & 0 & \Lambda_{22}^{-} & 0 & 0 \\
0 & 0 & 0 & -\left(\Lambda_{22}^{+}\right)^{-1} & 0 \\
0 & 0 & 0 & 0 & -\left(\Lambda_{22}^{-}\right)^{-1}
\end{array}\right]\left[\begin{array}{c}
w_{1} \\
X_{+}^{T} w_{2} \\
X_{-}^{T} w_{2} \\
X_{+}^{T} w_{3} \\
X_{-}^{T} w_{3}
\end{array}\right] d s
$$

We are now ready to answer the questions $i$ ) and ii) posed above. The boundary terms in the quadratic form (24) that can cause growth is equal to the sum of positive entries in $A_{11}, \Lambda_{22}^{+}$and $-\left(\Lambda_{22}^{-}\right)^{-1}$. The number of positive entries vary only with $A_{11}$ since the total number of positive entries in $\Lambda_{22}^{+}$and $-\left(\Lambda_{22}^{-}\right)^{-1}$ are constant and equal to the number of eigenvalues in $\tilde{A}_{22}$.

Remark 5. In the Navier-Stokes equations, $A_{11}=(\bar{u}, \bar{v}, \bar{w}) \cdot \hat{n}=u_{n}$, where $u_{n}$ is the outward pointing normal velocity on the boundary. Consequently, the Navier-Stokes equations require five boundary conditions at an inflow boundary $\left(u_{n}<0\right)$ and four at an outflow boundary $\left(u_{n}>0\right)$. This holds independently of whether the flow is subsonic or supersonic. The fact that the number of boundary conditions for the NavierStokes equations is independent of the speed of the flow, and only depends on the direction, is quite different from the situation for the Euler equations.

Remark 6. In the limit $\epsilon \rightarrow 0$ formally $w_{3}=F_{2}=\epsilon \tilde{F}_{2} \rightarrow 0$ in (24) and we are left with the number of boundary conditions for the Euler equations. ${ }^{5}$ However, $\tilde{F}_{2}$ contains gradients (see (3),(20)) and to this authors knowledge, this limit is not known. An analysis of the scalar viscous advection equation indicate that in fact $\epsilon \tilde{F}_{2} \neq 0$ as $\epsilon \rightarrow 0$. If this holds also for the Navier-Stokes equations, it means that the Euler equations are not the high Reynolds number approximation of the Navier-Stokes equations as commonly perceived.

\section{The form of the boundary conditions}

We proceed by splitting (24) into one positive and one negative part respectively

$$
\begin{aligned}
B T= & -\oint_{\delta \Omega}\left[\begin{array}{c}
\mathbf{1}_{+}\left(\gamma^{+}\right) w_{1} \\
X_{+}^{T} w_{2} \\
X_{-}^{T} w_{3}
\end{array}\right]^{T}\left[\begin{array}{ccc}
\gamma^{+} & 0 & 0 \\
0 & \Lambda_{22}^{+} & 0 \\
0 & 0 & -\left(\Lambda_{22}^{-}\right)^{-1}
\end{array}\right]\left[\begin{array}{c}
\mathbf{1}_{+}\left(\gamma^{+}\right) w_{1} \\
X_{+}^{T} w_{2} \\
X_{-}^{T} w_{3}
\end{array}\right] d s \\
& -\oint_{\delta \Omega}\left[\begin{array}{c}
\mathbf{1}_{-}\left(\gamma^{-}\right) w_{1} \\
X_{-}^{T} w_{2} \\
X_{+}^{T} w_{3}
\end{array}\right]^{T}\left[\begin{array}{ccc}
\gamma^{-} & 0 & 0 \\
0 & \Lambda_{22}^{-} & 0 \\
0 & 0 & -\left(\Lambda_{22}^{+}\right)^{-1}
\end{array}\right]\left[\begin{array}{c}
\mathbf{1}_{-}\left(\gamma^{-}\right) w_{1} \\
X_{-}^{T} w_{2} \\
X_{+}^{T} w_{3}
\end{array}\right] d s .
\end{aligned}
$$

In $(25), \mathbf{1}_{+}(x)$ and $\mathbf{1}_{-}(x)$ are indicator functions which are 1 if $x$ is positive or negative respectively, and zero otherwise. We have also used $\gamma^{+}=\left(A_{11}+\left|A_{11}\right|\right) / 2$ and $\gamma^{-}=\left(A_{11}-\left|A_{11}\right|\right) / 2$. To simplify the notation we introduce

$$
W^{+}=\left[\begin{array}{c}
\mathbf{1}_{+}\left(\gamma^{+}\right) w_{1} \\
X_{+}^{T} w_{2} \\
X_{-}^{T} w_{3}
\end{array} W^{-}=\left[\begin{array}{c}
\mathbf{1}_{-}\left(\gamma^{-}\right) w_{1} \\
X_{-}^{T} w_{2} \\
X_{+}^{T} w_{3}
\end{array}\right], \quad \Lambda^{-}=\left[\begin{array}{ccc}
\gamma^{+} & 0 & 0 \\
0 & \Lambda_{22}^{+} & 0 \\
0 & 0 & -\left(\Lambda_{22}^{-}\right)^{-1} \\
\gamma^{-} & 0 & 0 \\
0 & \Lambda_{22}^{-} & 0 \\
0 & 0 & -\left(\Lambda_{22}^{+}\right)^{-1}
\end{array}\right]\right. \text {. }
$$

Given the notations in (26), we rewrite (18) as

$$
\|U\|_{t}^{2}+2 D I_{c}=-\oint_{\delta \Omega}\left[\begin{array}{l}
W^{+} \\
W^{-}
\end{array}\right]^{T}\left[\begin{array}{cc}
\Lambda^{+} & 0 \\
0 & \Lambda^{-}
\end{array}\right]\left[\begin{array}{l}
W^{+} \\
W^{-}
\end{array}\right] d s .
$$

We are now ready to answer the question iii) posed above. Together with the previous answers to $i$-ii) we summarize the result in the following proposition. 
Proposition 1. The general form of the boundary condition in (16) that bound the right hand side of (27) (as well as (24)) and lead to well-posedness for zero boundary data or strong well-posedness for non-zero boundary data is

$$
W^{-}-R W^{+}=g .
$$

In (28), $R$ is a matrix with the number of rows equal to the number of boundary conditions and $g$ is given boundary data. The number of rows in $R$ is equal to the sum of positive entries in $A_{11}, \Lambda_{22}^{+}$and $-\left(\Lambda_{22}^{-}\right)^{-1}$ in (24) and vary only with the sign of $A_{11}$.

Proof. The number of positive entries in the matrix vary only with $A_{11}$ since the total number of positive entries in $\Lambda_{22}^{+}$and $-\left(\Lambda_{22}^{-}\right)^{-1}$ are constant and equal to the number of eigenvalues in $\tilde{A}_{22}$. The sign of $A_{11}$ vary with the direction of the normal $\hat{n}=\left(n_{1}, n_{2}, n_{3}\right)^{T}$ along the boundary $\delta \Omega$ since (20) holds. The proof that (28) bound (27) will be given below.

Remark 7. Note the close similarity of (28) with the way one imposes homogeneous boundary conditions for hyperbolic problems, where the ingoing characteristic variables are given by the outgoing ones and data.

\section{Weak and strong boundary conditions}

The boundary conditions in terms of $i$-iii) are now known and only one issue remains; they can be imposed weakly or strongly.

\section{Strongly imposed homogeneous boundary conditions}

The homogeneous version of the boundary condition (28) strongly imposed in (27) gives

$$
\|U\|_{t}^{2}+2 D I_{c}=-\oint_{\delta \Omega}\left(W^{+}\right)^{T}\left(R^{T} \Lambda^{-} R+\Lambda^{+}\right)\left(W^{+}\right) .
$$

To get a bound on the right-hand-side of (29), the matrix $R$ must satisfy

$$
R^{T} \Lambda^{-} R+\Lambda^{+} \geq 0 .
$$

Remark 8. Time-integration of (29) completes the proof of Proposition 1 for strongly imposed homogeneous boundary conditions and show that (see Definition 1) the problem (16) is well posed.

The general boundary operators used in (28) leading to an energy estimate and a well posed problem are

$$
H U=\left(H^{-}-R H^{+}\right) U .
$$

The operators $H^{+}$and $H^{-}$are decomposed as

$$
\begin{aligned}
& H^{+} U=\left(H_{0}^{+}+H_{D 0_{x}}^{+} \frac{\partial}{\partial x}+H_{D 0_{y}}^{+} \frac{\partial}{\partial y}+H_{D 0_{z}}^{+} \frac{\partial}{\partial z}\right) U=W^{+} \\
& H^{-} U=\left(H_{0}^{-}+H_{D 0_{x}}^{-} \frac{\partial}{\partial x}+H_{D 0_{y}}^{-} \frac{\partial}{\partial y}+H_{D 0_{z}}^{-} \frac{\partial}{\partial z}\right) U=W^{-}
\end{aligned}
$$

where

$$
\begin{aligned}
& H_{0}^{+}=\left[\begin{array}{cc}
\mathbf{1}_{+}\left(\gamma^{+}\right) & \mathbf{1}_{+}\left(\gamma^{+}\right)\left(A_{11}\right)^{-1} A_{12} \\
0 & X_{+}^{T} \\
0 & 0
\end{array}\right], \quad H_{0}^{-}=\left[\begin{array}{cc}
\mathbf{1}_{-}\left(\gamma^{-}\right) & \mathbf{1}_{-}\left(\gamma^{-}\right)\left(A_{11}\right)^{-1} A_{12} \\
0 & X_{-}^{T} \\
0 & 0
\end{array}\right] \\
& H_{D 0_{x}}^{+}=\left[\begin{array}{cc}
0 & 0 \\
0 & -X_{+}^{T}\left(\tilde{A}_{22}\right)^{-1} D_{1} \\
0 & X_{-}^{T} D_{1}
\end{array}\right], \quad H_{D 0_{x}}^{-}=\left[\begin{array}{cc}
0 & 0 \\
0 & -X_{-}^{T}\left(\tilde{A}_{22}\right)^{-1} D_{1} \\
0 & X_{+}^{T} D_{1}
\end{array}\right] \\
& H_{D 0_{y}}^{+}=\left[\begin{array}{cc}
0 & 0 \\
0 & -X_{+}^{T}\left(\tilde{A}_{22}\right)^{-1} D_{2} \\
0 & X_{-}^{T} D_{2}
\end{array}\right], \quad H_{D 0_{y}}^{-}=\left[\begin{array}{cc}
0 & 0 \\
0 & -X_{-}^{T}\left(\tilde{A}_{22}\right)^{-1} D_{2} \\
0 & X_{+}^{T} D_{2}
\end{array}\right] \\
& H_{D 0_{z}}^{+}=\left[\begin{array}{cc}
0 & 0 \\
0 & -X_{+}^{T}\left(\tilde{A}_{22}\right)^{-1} D_{3} \\
0 & X_{-}^{T} D_{3}
\end{array}\right], \quad H_{D 0_{z}}^{-}=\left[\begin{array}{cc}
0 & 0 \\
0 & -X_{-}^{T}\left(\tilde{A}_{22}\right)^{-1} D_{3} \\
0 & X_{+}^{T} D_{3}
\end{array}\right] .
\end{aligned}
$$


In (33), we denote $D_{1}, D_{2}$ and $D_{3}$ as

$$
D_{1}=n_{1} \bar{D}_{11}+n_{2} \bar{D}_{21}+n_{3} \bar{D}_{31}, \quad D_{2}=n_{1} \bar{D}_{12}+n_{2} \bar{D}_{22}+n_{3} \bar{D}_{32}, \quad D_{3}=n_{1} \bar{D}_{13}+n_{2} \bar{D}_{23}+n_{3} \bar{D}_{33}
$$

The boundary operators in (31)-(33) are obtained by combining (23), (26) and (28).

Remark 9. Strongly imposed boundary conditions are characterized by the fact that some of the variables in the boundary terms are replaced by others. In (29) for example, only $W^{+}$is present.

\section{Weakly imposed homogeneous boundary conditions}

By imposing the homogeneous boundary condition (28) weakly using (17), we obtain

$$
\|U\|_{t}^{2}+2 D I_{c}=-\oint_{\delta \Omega}\left[\begin{array}{l}
W^{+} \\
W^{-}
\end{array}\right]^{T}\left[\begin{array}{cc}
\Lambda^{+} & 0 \\
0 & \Lambda^{-}
\end{array}\right]\left[\begin{array}{l}
W^{+} \\
W^{-}
\end{array}\right]-U^{T} \Sigma\left(W^{-}-R W^{+}\right)-\left(U^{T} \Sigma\left(W^{-}-R W^{+}\right)\right)^{T} d s .
$$

By introducing $\Sigma^{-}$such that $U^{T} \Sigma=\left(W^{-}\right)^{T} \Sigma^{-},(35)$ becomes

$$
\|U\|_{t}^{2}+2 D I_{c}=\oint_{\delta \Omega}\left[\begin{array}{l}
W^{+} \\
W^{-}
\end{array}\right]^{T}\left[\begin{array}{cc}
\Lambda^{+} & R^{T}\left(\Sigma^{-}\right)^{T} \\
\Sigma^{-} R & \Lambda^{-}-\Sigma^{-}-\left(\Sigma^{-}\right)^{T}
\end{array}\right]\left[\begin{array}{l}
W^{+} \\
W^{-}
\end{array}\right] d s
$$

Remark 10. Weakly imposed boundary conditions are characterized by the fact that all variables are present in the boundary terms. In (36) for example, both $W^{+}$and $W^{-}$are present.

The choice $\Sigma^{-}=\Lambda^{-}$leads to $U^{T} \Sigma=\left(W^{-}\right)^{T} \Lambda^{-}$and the final penalty matrix

$$
\Sigma=\left(H^{-}\right)^{T} \Lambda^{-}
$$

By using (30), the energy rate (36) can now be written as

$$
\begin{aligned}
\|U\|_{t}^{2}+2 D I_{c} & =-\oint_{\delta \Omega}\left[\begin{array}{l}
W^{+} \\
W^{-}
\end{array}\right]^{T}\left[\begin{array}{cc}
\Lambda^{+} & R^{T} \Lambda^{-} \\
\Lambda^{-} R & -\Lambda^{-}
\end{array}\right]\left[\begin{array}{l}
W^{+} \\
W^{-}
\end{array}\right] d s \\
& =-\oint_{\delta \Omega}\left(W^{+}\right)^{T}\left(R^{T} \Lambda^{-} R+\Lambda^{+}\right)\left(W^{+}\right)+\left[\begin{array}{l}
W^{+} \\
W^{-}
\end{array}\right]^{T}\left[\begin{array}{cc}
-R^{T} \Lambda^{-} R & R^{T} \Lambda^{-} \\
\Lambda^{-} R & -\Lambda^{-}
\end{array}\right]\left[\begin{array}{l}
W^{+} \\
W^{-}
\end{array}\right] d s \\
& =-\oint_{\delta \Omega}\left(W^{+}\right)^{T}\left(R^{T} \Lambda^{-} R+\Lambda^{+}\right)\left(W^{+}\right)-\left(W^{-}-R W^{+}\right)^{T} \Lambda^{-}\left(W^{-}-R W^{+}\right) d s
\end{aligned}
$$

where the right-hand-side is negative semi-definite.

Remark 11. Time-integration of (38) completes the proof of Proposition 1 for weakly imposed homogeneous boundary conditions and show that (see Definition 1) the problem (17) is well posed.

Remark 12. The energy estimate (38) shows that a weak imposition of well-posed homogeneous boundary conditions produces the strong energy rate with an additional term $-\left(W^{-}-R W^{+}\right)^{T} \Lambda^{-}\left(W^{-}-R W^{+}\right)$that is proportional to the boundary condition. A similar, dissipative term will appear in the discrete approximation.

We can now prove

Proposition 2. The weak and strong solutions to (17) and (16) with homogeneous boundary conditions are identical. The boundary conditions are satisfied exactly also in the weak formulation

Proof. The energy estimate (38) implies uniqueness. Since the strong solution satisfies the weak formulation, the strong and weak solution are identical. Consequently, the boundary conditions are satisfied exactly also in the weak procedure, and the additional term in the estimate (38) is identically zero. 


\section{Strongly imposed non-homogeneous boundary conditions}

The boundary conditions (28) strongly imposed in (27) leads to

$$
\|U\|_{t}^{2}+2 D I_{c}=-\oint_{\delta \Omega}\left[\begin{array}{c}
W^{+} \\
g
\end{array}\right]^{T}\left[\begin{array}{cc}
R^{T} \Lambda^{-} R+\Lambda^{+} & R^{T} \Lambda^{-} \\
\Lambda^{-} R & \Lambda^{-}
\end{array}\right]\left[\begin{array}{c}
W^{+} \\
g
\end{array}\right] d s .
$$

We can now add and subtract $g^{T} G g$ where $G$ is a positive semi-definite bounded matrix ${ }^{22}$ to obtain

$$
\|U\|_{t}^{2}+2 D I_{c}=-\oint_{\delta \Omega}\left[\begin{array}{c}
W^{+} \\
g
\end{array}\right]^{T}\left[\begin{array}{cc}
R^{T} \Lambda^{-} R+\Lambda^{+} & R^{T} \Lambda^{-} \\
\Lambda^{-} R & G
\end{array}\right]\left[\begin{array}{c}
W^{+} \\
g
\end{array}\right]+g^{T}\left(-G+\Lambda^{-}\right) g d s .
$$

The choice

$$
G \geq\left(\Lambda^{-} R\right)\left(R^{T} \Lambda^{-} R+\Lambda^{+}\right)^{-1}\left(\Lambda^{-} R\right)^{T}
$$

bounds the right-hand-side of (40). In order for condition (41) to make sense, we need to sharpen (30) to

$$
R^{T} \Lambda^{-} R+\Lambda^{+}>0
$$

Remark 13. Time-integration of (40) completes the proof of Proposition 1 for strongly imposed nonhomogeneous boundary conditions and show that (see Definition 2) the problem (16) is strongly well posed.

Remark 14. If (42) holds, then the choice (41) can always be made, and we can estimate the solution in terms of the boundary data which leads to a strongly well-posed problem. If condition (30) holds, but not (42), we get an energy estimate for zero boundary data and we have a well posed problem. ${ }^{6}$ Note also that even if $\Lambda^{+}$is singular, which is not the case for the Navier-Stokes equations, $G$ can be chosen in a similar way as in (41) by separating out the zero eigenvalues.

\section{Weakly imposed non-homogeneous boundary conditions}

The boundary conditions (28) imposed weakly using (17) yields

$$
\|U\|_{t}^{2}+2 D I_{c}=-\oint_{\delta \Omega}\left[\begin{array}{l}
W^{+} \\
W^{-}
\end{array}\right]^{T}\left[\begin{array}{cc}
\Lambda^{+} & 0 \\
0 & \Lambda^{-}
\end{array}\right]\left[\begin{array}{l}
W^{+} \\
W^{-}
\end{array}\right]+U^{T} \Sigma\left(W^{-}-R W^{+}-g\right)+\left(U^{T} \Sigma\left(W^{-}-R W^{+}-g\right)\right)^{T} d s
$$

where $\Sigma$ is the penalty matrix. Following the analysis above, we choose $\Sigma$ such that $U^{T} \Sigma=-\left(W^{-}\right)^{T} \Lambda^{-}$, and insert this into (43) to find

$$
\|U\|_{t}^{2}+2 D I_{c}=-\oint_{\delta \Omega}\left[\begin{array}{c}
W^{+} \\
W^{-} \\
g
\end{array}\right]^{T} \underbrace{\left[\begin{array}{ccc}
\Lambda^{+} & R^{T} \Lambda^{-} & 0 \\
\Lambda^{-} R & -\Lambda^{-} & \Lambda^{-} \\
0 & \Lambda^{-} & 0
\end{array}\right]}_{M}\left[\begin{array}{c}
W^{+} \\
W^{-} \\
g
\end{array}\right] d s .
$$

The matrix $M$ in (44) can be divided into three parts and rewritten as

$$
M=\left[\begin{array}{ccc}
-R^{T} \Lambda^{-} R & R^{T} \Lambda^{-} & -R^{T} \Lambda^{-} \\
\Lambda^{-} R & -\Lambda^{-} & \Lambda^{-} \\
-\Lambda^{-} R & \Lambda^{-} & -\Lambda^{-}
\end{array}\right]+\left[\begin{array}{ccc}
R^{T} \Lambda^{-} R+\Lambda^{+} & 0 & R^{T} \Lambda^{-} \\
0 & 0 & 0 \\
\Lambda^{-} R & 0 & G
\end{array}\right]+\left[\begin{array}{ccc}
0 & 0 & 0 \\
0 & 0 & 0 \\
0 & 0 & -G+\Lambda^{-}
\end{array}\right]
$$

The second matrix above is positive semi-definite by the choice of $G$ in (41), while the third matrix is bounded by data. These two matrices correspond exactly to the result obtained for strong boundary conditions.

The first matrix in (45), which is due to the use of weak boundary conditions, can be rewritten as

$$
\left[\begin{array}{ccc}
R & 0 & 0 \\
0 & I & 0 \\
0 & 0 & I
\end{array}\right]^{T}\left(C_{0} \otimes \Lambda^{-}\right)\left[\begin{array}{ccc}
R & 0 & 0 \\
0 & I & 0 \\
0 & 0 & I
\end{array}\right], \quad C_{0}=\left[\begin{array}{ccc}
-1 & +1 & -1 \\
+1 & -1 & +1 \\
-1 & +1 & -1
\end{array}\right]
$$


where $\otimes$ denotes the Kronecker product. ${ }^{27}$ The matrix $C_{0}$ is negative semi-definite with eigenvalues $-3,0,0$ and hence the right-hand-side of (44) is bounded by data.

The difference between the estimate (40) obtained by strong imposition of boundary conditions and the estimate (44) obtained by a weak imposition is the term

$$
\tilde{R}=-\oint_{\delta \Omega}\left[\begin{array}{c}
W^{+} \\
W^{-} \\
g
\end{array}\right]^{T}\left[\begin{array}{ccc}
R & 0 & 0 \\
0 & I & 0 \\
0 & 0 & I
\end{array}\right]^{T}\left(C_{0} \otimes \Lambda^{-}\right)\left[\begin{array}{ccc}
R & 0 & 0 \\
0 & I & 0 \\
0 & 0 & I
\end{array}\right]\left[\begin{array}{c}
W^{+} \\
W^{-} \\
g
\end{array}\right] d s
$$

on the right-hand-side in the weak energy rate. We can expand the term $\tilde{R}$ by using

$$
C_{0}=X \Gamma X^{T}, \quad X=\frac{1}{\sqrt{3}}\left[\begin{array}{ccc}
-1 & 1 & 1 \\
1 & 1 & -1 \\
-1 & 0 & -2
\end{array}\right], \quad \Gamma=\left[\begin{array}{ccc}
-3 & 0 & 0 \\
0 & 0 & 0 \\
0 & 0 & 0
\end{array}\right]
$$

and find

$$
\begin{aligned}
\tilde{R} & =-\oint_{\delta \Omega}\left[\begin{array}{c}
R W^{+} \\
W^{-} \\
g
\end{array}\right]^{T}\left(X \Gamma X^{T} \otimes \Lambda^{-}\right)\left[\begin{array}{c}
R W^{+} \\
W^{-} \\
g
\end{array}\right] d s \\
& =-\oint_{\delta \Omega}\left[\begin{array}{c}
W^{-}-R W^{+}-g \\
R W^{+}+W^{-} \\
R W^{+}-W^{-}+2 g
\end{array}\right]^{T}\left(\left[\begin{array}{ccc}
-1 & 0 & 0 \\
0 & 0 & 0 \\
0 & 0 & 0
\end{array}\right] \otimes \Lambda^{-}\right)\left[\begin{array}{c}
W^{-}-R W^{+}-g \\
R W^{+}+W^{-} \\
R W^{+}-W^{-}+2 g
\end{array}\right] d s \\
& =+\oint_{\delta \Omega}\left(W^{+}-R W^{-}-g\right)^{T} \Lambda^{-}\left(W^{+}-R W^{-}-g\right) d s \leq 0 .
\end{aligned}
$$

Remark 15. Time-integration of (44) completes the proof of Proposition 1 for weakly imposed non-homogeneous boundary conditions and show that (see Definition 2) the problem (17) is strongly well posed.

Remark 16. Just as in the case for homogeneous boundary conditions, the additional term $R=\oint_{\delta \Omega}\left(W^{-}-\right.$ $\left.R W^{+}-g\right)^{T} \Lambda^{-}\left(W^{-}-R W^{+}-g\right)$ in the weak energy rate is proportional to the boundary condition. A similar dissipative term will appear in the discrete approximation.

We can also for the non-homogeneous case prove

Proposition 3. The weak and strong solutions to (17) and (16) with non-homogeneous boundary conditions are identical. The boundary conditions are satisfied exactly also in the weak formulation.

Proof. The proof is identical to the one given in the homogeneous case for Proposition 2.

\section{The semi-discrete approximation}

Consider the semi-discrete approximation of (16) on a cubic domain with weakly imposed boundary conditions

$$
\begin{aligned}
V_{t} & +\left(D_{x} \otimes I_{y} \otimes I_{z} \otimes A\right) V+\left(I_{x} \otimes D_{y} \otimes I_{z} \otimes B\right) V+\left(I_{x} \otimes I_{y} \otimes D_{z} \otimes C\right) V \\
& =\left(D_{x} \otimes I_{y} \otimes I_{z} \otimes I_{M}\right) F+\left(I_{x} \otimes D_{y} \otimes I_{z} \otimes I_{M}\right) G+\left(I_{x} \otimes I_{y} \otimes D_{z} \otimes I_{M}\right) H \\
& +\left(E_{N} P_{x}^{-1} \Sigma \otimes I_{y} \otimes I_{z} \otimes I_{M}\right)\left(\left(\tilde{H}^{+}-\tilde{R} \tilde{H}^{-}\right) V-e_{N_{x}} \otimes g\right) \\
V(0) & =f .
\end{aligned}
$$

The discrete solution $V_{i j k}(t) \approx u\left(x_{i}, y_{j}, z_{k}, t\right)$ is arranged as

$$
V=\left[\begin{array}{c}
V_{0} \\
V_{1} \\
\vdots \\
V_{i} \\
\vdots \\
V_{N_{x}}
\end{array}\right], \quad V_{i}=\left[\begin{array}{c}
V_{0} \\
V_{1} \\
\vdots \\
V_{j} \\
\vdots \\
V_{N_{y}}
\end{array}\right]_{i} \quad V_{j}=\left[\begin{array}{c}
V_{0} \\
V_{1} \\
\vdots \\
V_{k} \\
\vdots \\
V_{N_{z}}
\end{array}\right]_{i j}
$$


The matrices $\bar{A}, \bar{B}$ and $\bar{C}$ are matrices given in (16). For simplicity, only the boundary condition at $x=1$ is considered by inserting the penalty term (corresponding to the lifting operator $L$ in (17)) only at $k=N$. The discrete representation of the vectors $\bar{F}, \bar{G}$ and $\bar{H}$ in (3) are

$$
\begin{aligned}
\tilde{F} & =\left(\tilde{I} \otimes \bar{D}_{11}\right) V_{x}+\left(\tilde{I} \otimes \bar{D}_{12}\right) V_{y}+\left(\tilde{I} \otimes \bar{D}_{13}\right) V_{z} \\
\tilde{G} & =\left(\tilde{I} \otimes \bar{D}_{21}\right) V_{x}+\left(\tilde{I} \otimes \bar{D}_{22}\right) V_{y}+\left(\tilde{I} \otimes \bar{D}_{23}\right) V_{z} \\
\tilde{H} & =\left(\tilde{I} \otimes \bar{D}_{31}\right) V_{x}+\left(\tilde{I} \otimes \bar{D}_{32}\right) V_{y}+\left(\tilde{I} \otimes \bar{D}_{33}\right) V_{z},
\end{aligned}
$$

where we, with a slight abuse of notation, used $\tilde{I}=\left(I_{x} \otimes I_{y} \otimes I_{z}\right)$ and

$$
V_{x}=\left(D_{x} \otimes I_{y} \otimes I_{z} \otimes I_{M}\right) V, \quad V_{y}=\left(I_{y} \otimes D_{y} \otimes I_{z} \otimes I_{M}\right) V, \quad V_{z}=\left(I_{z} \otimes I_{y} \otimes D_{z} \otimes I_{M}\right) V .
$$

The difference operators are on summation-by-parts form (SBP), ${ }^{23}$ i.e. $D_{x, y, z}=P_{x, y, z}^{-1} Q_{x, y, z}$ where $P_{x, y, z}=P_{x, y, z}^{T}>0, Q_{x, y, z}+Q_{x, y, z}^{T}=\operatorname{diag}(-1,0 \ldots, 0,+1), E_{N}$ a zero matrix where the element $(N+1, N+1)$ is one. $I_{x}, I_{y}, I_{z}$ and $I_{M}$ are identity matrices of appropriate sizes and $e_{N_{x}}=[0, \ldots 0,1]$ of length $N_{x}$.

The continuous boundary operator in (31) is $H^{-}-R H^{+}$where both $H^{+}$and $H^{-}$are partioned matrix operators of Robin type, see (33). To construct the corresponding discrete operators we use that partitioning and define the discrete versions of $H^{+}$and $H^{-}$as

$$
\begin{aligned}
\tilde{H}^{+} & =\left(I_{x} \otimes I_{y} \otimes I_{z} \otimes H_{0}^{+}\right)+\left(D_{x} \otimes I_{y} \otimes I_{z} \otimes H_{D 0 x}^{+}\right) \\
& +\left(I_{x} \otimes D_{y} \otimes I_{z} \otimes H_{D 0 y}^{+}\right)+\left(I_{x} \otimes I_{y} \otimes D_{z} \otimes H_{D 0 z}^{+}\right), \\
\tilde{H}^{-} & =\left(I_{x} \otimes I_{y} \otimes I_{z} \otimes H_{0}^{-}\right)+\left(D_{x} \otimes I_{y} \otimes I_{z} \otimes H_{D 0 x}^{-}\right) \\
& +\left(I_{x} \otimes D_{y} \otimes I_{z} \otimes H_{D 0 y}^{-}\right)+\left(I_{x} \otimes I_{y} \otimes D_{z} \otimes H_{D 0 z}^{-}\right) .
\end{aligned}
$$

\section{A. The energy method}

We mimic the analysis of the continuous problem above, but limit ourselves to weak boundary conditions.

\section{Weakly imposed homogeneous boundary conditions}

The discrete energy method (multiply with $V^{T}\left(P_{x} \otimes P_{y} \otimes P_{z} \otimes I_{M}\right)$ from the left and add the transpose) applied to (50) with $g=0$ gives

$$
\begin{aligned}
\frac{d}{d t}\|V\|_{P_{x y z}}^{2}+2 D I_{d}= & -V^{T}\left(E_{N} \otimes P_{y} \otimes P_{z} \otimes A\right) V+V^{T}\left(E_{N} \otimes P_{y} \otimes P_{z} \otimes I_{M}\right) \tilde{F} \\
& +\tilde{F}^{T}\left(E_{N} \otimes P_{y} \otimes P_{z} \otimes I_{M}\right) V+V^{T} \tilde{\Sigma}\left(E_{N} \otimes P_{y} \otimes P_{z} \otimes I_{M}\right)\left(\tilde{H}^{-}-\tilde{R} \tilde{H}^{+}\right) V \\
& +V^{T}\left(\tilde{H}^{-}-\tilde{R} \tilde{H}^{+}\right)^{T}\left(E_{N} \otimes P_{y} \otimes P_{z} \otimes I_{M}\right) \tilde{\Sigma}^{T} V
\end{aligned}
$$

where

$$
\begin{aligned}
D I_{d} & =\left[\begin{array}{c}
V_{x} \\
V_{y} \\
V_{z}
\end{array}\right]^{T} P_{x y z}\left[\begin{array}{ccc}
\tilde{I} \otimes \bar{D}_{11} & \tilde{I} \otimes \bar{D}_{12} & \tilde{I} \otimes \bar{D}_{13} \\
\tilde{I} \otimes \bar{D}_{21} & \tilde{I} \otimes \bar{D}_{22} & \tilde{I} \otimes \bar{D}_{23} \\
\tilde{I} \otimes \bar{D}_{31} & \tilde{I} \otimes \bar{D}_{32} & \tilde{I} \otimes \bar{D}_{33}
\end{array}\right]\left[\begin{array}{c}
V_{x} \\
V_{y} \\
V_{z}
\end{array}\right] \\
= & {\left[\begin{array}{c}
V_{x} \\
V_{y} \\
V_{z}
\end{array}\right]^{T} P_{x y z}\left(\Psi^{T}\left(\left[\begin{array}{ccc}
\bar{D}_{11} & \bar{D}_{12} & \bar{D}_{13} \\
\bar{D}_{21} & \bar{D}_{22} & \bar{D}_{23} \\
\bar{D}_{31} & \bar{D}_{32} & \bar{D}_{33}
\end{array}\right] \otimes \tilde{I}\right) \Psi\right)\left[\begin{array}{c}
V_{x} \\
V_{y} \\
V_{z}
\end{array}\right]>0 . }
\end{aligned}
$$

In (56), we have used that the Kronecker product ${ }^{27}$ is even permutation similar for square matrices. Note that $D I_{d}$ mimics the continuous dissipation $D I_{c}$ and it is clearly positive semi-definite. We have also used the notation $P_{x y z}=\left(P_{x} \otimes P_{y} \otimes P_{z} \otimes I_{M}\right)$ and $\tilde{R}=(\tilde{I} \otimes R)$.

Recall that $\left(H^{-}-R H^{+}\right) U=W^{-}-R W^{+}$in the continuous case. The corresponding discrete relation reads

$$
\begin{aligned}
\left(\tilde{H}^{-}-\tilde{R} \tilde{H}^{+}\right) V & =\left[\left(\tilde{I} \otimes H_{0}^{-}\right) V+\left(\tilde{I} \otimes H_{D 0 x}^{-}\right) V_{x}+\left(\tilde{I} \otimes H_{D 0 y}^{-}\right) V_{y}+\left(\tilde{I} \otimes H_{D 0 z}^{-}\right) V_{z}\right] \\
& -\tilde{R}\left[\left(\tilde{I} \otimes H_{0}^{+}\right) V+\left(\tilde{I} \otimes H_{D 0 x}^{+}\right) V_{x}+\left(\tilde{I} \otimes H_{D 0 y}^{+}\right) V_{y}+\left(\tilde{I} \otimes H_{D 0 z}^{+}\right) V_{z}\right] \\
& =\tilde{W}^{-}-\tilde{R} \tilde{W}^{+} .
\end{aligned}
$$


By expanding the fluxes defined in (52) and subsequently diagonalizing the resulting matrix, we obtain

$$
\begin{aligned}
\frac{d}{d t}\|V\|_{P_{x y z}}^{2}+2 D I_{d}= & -\left[\begin{array}{c}
\tilde{W}^{+} \\
\tilde{W}^{-}
\end{array}\right]_{N}^{T}\left(P_{y z} \otimes\left[\begin{array}{cc}
\Lambda^{+} & 0 \\
0 & \Lambda^{-}
\end{array}\right]\right)\left[\begin{array}{c}
\tilde{W}^{+} \\
\tilde{W}^{-}
\end{array}\right]_{N} \\
& +V^{T} \tilde{\Sigma}\left(E_{N} \otimes P_{y} \otimes P_{z} \otimes I_{M}\right)\left(\tilde{W}^{-}-\tilde{R} \tilde{W}^{+}\right) \\
& +\left(\tilde{W}^{-}-\tilde{R} \tilde{W}^{+}\right)^{T}\left(E_{N} \otimes P_{y} \otimes P_{z} \otimes I_{M}\right) \tilde{\Sigma}^{T} V
\end{aligned}
$$

which is the discrete version of (35). In (58), $P_{y z}$ denotes $P_{y} \otimes P_{z}$.

To mimic the continuous setting we let $V^{T} \tilde{\Sigma}=\left(\tilde{W}^{-}\right)^{T} \tilde{\Sigma}^{-}$which implies $\tilde{\Sigma}=\left(\tilde{H}^{-}\right)^{T} \tilde{\Sigma}^{-}$. The additional choice $\tilde{\Sigma}^{-}=\left(\tilde{I} \otimes \Sigma^{-}\right)$gives

$$
\frac{d}{d t}\|V\|_{P_{x y z}}^{2}+2 D I_{d}=-\left[\begin{array}{c}
\tilde{W}^{+} \\
\tilde{W}^{-}
\end{array}\right]_{N}^{T}\left(P_{y z} \otimes\left[\begin{array}{cc}
\Lambda^{+} & R^{T} \tilde{\Sigma}^{-} \\
\left(\tilde{\Sigma}^{-}\right)^{T} R & \Lambda^{-}-\tilde{\Sigma}^{-}-\left(\tilde{\Sigma}^{-}\right)^{T}
\end{array}\right]\right)\left[\begin{array}{l}
\tilde{W}^{+} \\
\tilde{W}^{-}
\end{array}\right]_{N}
$$

which corresponds to (36) in the continuous case. As in the continuous case we let $\Sigma^{-}=\Lambda^{-}$which yields

$$
\tilde{\Sigma}=\left(\tilde{H}^{-}\right)^{T}\left(\tilde{I} \otimes \Lambda^{-}\right)
$$

corresponding to (37) and the energy rate

$$
\frac{d}{d t}\|V\|_{P_{x y z}}^{2}+2 D I_{d}=-\left(\tilde{W}_{N}^{+}\right)^{T}\left(P_{y z} \otimes\left(R^{T} \Lambda^{-} R+\Lambda^{+}\right)\right)\left(\tilde{W}_{N}^{+}\right)+\left(\tilde{W}_{N}^{-}-R \tilde{W}_{N}^{+}\right)^{T}\left(P_{y z} \otimes \Lambda^{-}\right)\left(\tilde{W}_{N}^{-}-R \tilde{W}_{N}^{+}\right)
$$

which correspond to (38). The second term in (61) which was zero in the continuous case, now adds a small amount of dissipation.

We can summarize the result in the following Proposition.

Proposition 4. The semi-discrete approximation (50) of (16) with homogeneous weak boundary conditions and penalty matrix (60) is stable.

Proof. Time-integration of (61) lead to an estimate of the form (14) given in Definition 3.

By the fact that the semi-discrete energy rate (61) mimics the continuous energy rate (38) term by term and will converge to the continuous solution, we can also state

Proposition 5. The semi-discrete approximation (50) of (16) and penalty matrix (60) is strictly stable.

Remark 17. The derivation in this section is completely analogous to the continuous one above. In fact, the boundary conditions and penalty matrices are already derived in the analysis of the continuous problem.

\section{Weakly imposed non-homogeneous boundary conditions}

By using the same procedure as for the homogeneous case but with non-zero data, we end up with

$$
\frac{d}{d t}\|V\|_{P_{x y z}}^{2}+2 D I_{d}=-\left[\begin{array}{c}
\tilde{W}^{+} \\
\tilde{W}^{-} \\
g
\end{array}\right]_{N}^{T}(\underbrace{\left[\begin{array}{ccc}
\Lambda^{+} & R^{T} \Lambda^{-} & 0 \\
\Lambda^{-} R & -\Lambda^{-} & \Lambda^{-} \\
0 & \Lambda^{-} & 0
\end{array}\right]}_{M})\left[\begin{array}{c}
\tilde{W}^{+} \\
\tilde{W}^{-} \\
g
\end{array}\right]_{N}
$$

where $M$ in (62) is exactly the same matrix as in (44). Consequently, the continuous analysis leads directly to strong stability. The discrete energy estimate is similar to the continuous one but the additional term

$$
\begin{gathered}
{\left[\begin{array}{c}
\tilde{W}^{+} \\
\tilde{W}^{-} \\
g
\end{array}\right]_{N}^{T}\left(P_{y z} \otimes\left[\begin{array}{ccc}
-R^{T} \Lambda^{-} R & R^{T} \Lambda^{-} & -R^{T} \Lambda^{-} \\
\Lambda^{-} R & -\Lambda^{-} & \Lambda^{-} \\
-\Lambda^{-} R & \Lambda^{-} & -\Lambda^{-}
\end{array}\right]\right)\left[\begin{array}{c}
\tilde{W}^{+} \\
\tilde{W}^{-} \\
g
\end{array}\right]_{N}=} \\
-\quad\left(\tilde{W}_{N}^{-}-R \tilde{W}_{N}^{+}-\tilde{g}_{N}\right)^{T}\left(P_{y z} \otimes \Lambda^{-}\right)\left(\tilde{W}_{N}^{-}-R_{N} \tilde{W}_{N}^{+}-\tilde{g}_{N}\right)
\end{gathered}
$$

which was zero in the continuous case, now adds a small amount of dissipation.

We can summarize the result in the following Proposition. 
Proposition 6. The semi-discrete approximation (50) of (16) with non-homogeneous weak boundary condtions and penalty matrix (60) is strongly stable.

Proof. Time-integration of (62) lead to an estimate of the form (15) given in Definition 4.

Remark 18. Just as in the preceding section on weak homogeneous boundary conditions, the derivation in the semi-discrete case is completely analogous to the continuous one above.

\section{Conclusions}

We have analyzed the time-dependent compressible Navier-Stokes equations, and in particular the role of boundary conditions. The number of boundary conditions, where to impose them and their form have been derived. The procedure is based on the energy method and generalize the characteristic boundary procedure for hyperbolic problems like the Euler equations.

The boundary conditions can be imposed weakly or strongly. It was shown that the weak and strong boundary procedures produce identical solutions and that the boundary conditions are satisfied exactly also in the weak procedure. It was shown that the derived boundary conditions lead to strongly well posed problems both for the weak and strong imposition.

It was also shown that the weak boundary procedures in the well-posedness analysis lead directly to stability, strong stability and strict stability of the numerical approximation. The boundary conditions and penalty matrices were already derived in the analysis of the continuous problem. Almost no additional derivations were necessary.

The analysis of the time-dependent compressible Navier-Stokes equations that was done in this paper is completely general. It can be extended to any coupled system of partial differential equations posed as an initial boundary value problem without difficulty.

\section{References}

${ }^{1}$ J. S. Hesthaven and D. Gottlieb, A stable penalty method for the compressible Navier-Stokes equations: I. Open boundary conditions, SIAM J. Sci. Comput., 17 (1996), pp. 579612.

${ }^{2}$ J. Nordström, The influence of open boundary conditions on the convergence to steady state for the NavierStokes equations, J. Comput. Phys., 85 (1989), pp. 210244.

${ }^{3} \mathrm{~J}$. Nordström, The use of characteristic boundary conditions for the Navier-Stokes equations, Computers and Fluids, 24 (1995), pp. 609623.

${ }^{4}$ B. Gustafsson and A. Sundström, Incompletely parabolic systems in fluid dynamics, SIAM J. Appl. Math., 35 (1978), pp. 343357.

${ }^{5}$ J. Nordström and M. Svärd, Well Posed Boundary Conditions for the Navier-Stokes Equation, SIAM Journal on Numerical Analysis, 43, (2005), pp. 1231-1255.

${ }^{6}$ B. Gustafsson, H.-O. Kreiss, and J. Oliger, Time Dependent Problems and Difference Methods, John Wiley and Sons, New York, 1995.

${ }^{7}$ H.-O. Kreiss, Initial boundary value problems for hyperbolic systems, Comm. Pure Appl. Math., 23, (1970), pp. 277-298.

${ }^{8}$ J. C. Strikwerda, Initial boundary value problems for incompletely parabolic systems, Comm. Pure Appl. Math., 30 (1977), pp. $797-822$.

${ }^{9}$ S. V. Tsynkov, Numerical solution of problems on unbounded domains. A review, Appl. Numer. Math., 27 (1998), pp. 465532 .

${ }^{10}$ D. Givoli, High-order local non-reflecting boundary conditions: a review, Wave Motion, 39, (2004), pp. 319326.

${ }^{11}$ M. H. Carpenter, J. Nordström and D. Gottlieb, A Stable and Conservative Interface Treatment of Arbitrary Spatial Accuracy, Journal of Computational Physics, 148, (1999), pp. 341-365.

${ }^{12}$ M. Svärd, M. H. Carpenter and J. Nordström, A Stable High-Order Finite Difference Scheme for the Compressible Navier-Stokes Equations, far-field boundary conditions, Journal of Computational Physics, 225, (2007), pp. 1020-1038.

${ }^{13}$ J. Nordström, K. Forsberg, C. Adamsson and P. Eliasson, Finite Volume Methods, Unstructured Meshes and Strict Stability, Applied Numerical Mathematics, 48, (2003), pp. 453-473.

${ }^{14}$ J. Nordström, S. Eriksson and P. Eliasson, Weak and Strong Wall Boundary Procedures and Convergence to Steady-State of the Navier-Stokes Equations, Journal of Computational Physics, 231, (2012), pp. 4867-4884.

${ }^{15}$ M.H. Carpenter, D. Gottlieb, Spectral methods on arbitrary grids, Journal of Computational Physics, 129, (1996), pp. 74-86.

${ }^{16}$ M.H. Carpenter, T.C. Fisher, E.J. Nielsen and S.H. Frankel, Entropy stable spectral collocation schemes for the NavierStokes Equations: Discontinuous interfaces, SIAM Journal on Scientific Computing, 36, (2014), pp. B835-B867.

${ }^{17}$ G.J. Gassner, A skew-symmetric discontinuous Galerkin spectral element discretization and its relation to sbp-sat finite difference methods, SIAM Journal on Scientific Computing, 35, (2013), pp. A1233-A1253. 
${ }^{18} \mathrm{H}$. Huynh, A flux reconstruction approach to high-order schemes including discontinuous Galerkin methods, 18th AIAA Computational Fluid Dynamics Conference, Miami, FL, Jun 25-28, 2007.

${ }^{19}$ P. Castonguay, D.M Williams, P.E. Vincent and A.J. Jameson, Energy stable flux reconstruction schemes for advectiondiffusion problems, Computer Methods in Applied Mechanics and Engineering, 267, (2013), pp. 400-417.

${ }^{20} \mathrm{~S}$. Abarbanel and D. Gottlieb, Optimal time splitting for two- and three-dimensional Navier-Stokes equations with mixed derivatives, Journal of Computational Physics, 41, (1981), pp. 1-33.

${ }^{21}$ J. Nordström and B. Lönn, Energy Decay of Vortices in Viscous Fluids: an Applied Mathematics View, Journal of Fluid Mechanics, 709, (2012), pp. 593609.

${ }^{22} \mathrm{~J}$. Nordström \& M. Wahlsten, Variance reduction through robust design of boundary conditions for stochastic hyperbolic systems of equations, Journal of Computational Physics, 82, (2015), pp. 1-22.

${ }^{23}$ M. Svärd and J. Nordström, Review of Summation-By-Parts Schemes for Initial-Boundary-Value Problems, Journal of Computational Physics, 268, (2014), pp. 1738.

${ }^{24}$ K. Mattson and J. Nordström, Summation by parts operators for finite difference approximations of second derivatives, Journal of Computational Physics, 199, (2004), pp. 503-540.

${ }^{25}$ J.J. Sudirham, J.J. W. van der Vegt and R.M.J. van Damme, A study on discontinuous Galerkin finite element methods for eliptic problems, Memorandum 1690, Faculty of EEMCS, University of Twente, September 2003.

${ }^{26}$ D.N. Arnold, F. Brezzi, B. Cockburn and L. Donatella Marini, Unified analysis of discontinuous Galerkin methods for elliptic problems, SIAM Journal on Numerical Analysis, 39, (2001), pp. 1749-1779.

${ }^{27}$ R.A. Horn and C.R. Johnson, Topics in Matrix Analysis, Cambridge University Press, 1991. 